\title{
Interfacial Adhesion Strength between Fibers of Liquid Crystalline Polymer and Thermoplastic Matrix
}

\author{
Kilwon Cho, ${ }^{\dagger}$ Taehyun Kong, and Daeho LeE \\ Department of Chemical Engineering, Pohang University of Science and Technology, \\ Pohang 790-784, Korea
}

(Received March 21, 1997)

\begin{abstract}
The interfacial shear stress between fibers of liquid crystalline polymer (LCP) and various thermoplastics was evaluated using a single fiber composite test. Several ductile polymers such as polycarbonate (PC), polyethersulfone (PSF), and polyetherimide (PEI) were employed as a matrix polymer. The effects of matrix properties, welding time and welding temperature on the interfacial shear stress were examined. The interfacial shear stress increased abruptly at the initial welding time and became an equilibrium value, which implies that the wetting approached the equilibrium with increasing welding time. The equilibrium interfacial shear stress, $\tau$ decreased in the order of PSF/LCP, PEI/LCP, and PC/LCP. The debonding occurred at the interface and the plastic deformation of the matrix was not observed in the case of PC/LCP. However, the plastic deformation of the matrix near the interface was observed in the case of PSF/LCP. Through the measurement of polarity and compressive pressure, the polar interaction and compressive pressure were found to be the main interfacial adhesion mechanisms between LCP fibers and thermoplastic matrices.

KEY WORDS Liquid Crystalline Polymer / Interfacial Adhesion / Single Fiber Composite Test / Thermoplastic /
\end{abstract}

The most representative method using thermotropic liquid crystalline polymer (TLCP) is manufacturing in situ composite through melt processing of TLCP/thermoplastics blends. ${ }^{1,2}$ In this case, the TLCP droplets in the blend are drawn in the direction of spinning or injection molding during processing and the microfibrils are developed in the matrix polymer. The mechanical properties of in situ composite containing TLCP are affected by TLCP content, microfibrillation of TLCP, and the interfacial adhesion between TLCP fiber and matrix polymer. $^{3}$ Many studies on the mechanical properties of in situ composite containing TLCP have been done using various thermoplastics. However, most of these studies are concentrated on the effect of TLCP content and microfibrillation of TLCP on the mechanical properties of in situ composites. ${ }^{4-7}$

It is known that the stress transfer from the matrix polymer to the reinforcing fiber depends on the interfacial adhesion between reinforcing fiber and matrix polymer and it affects the mechanical properties of composite materials. ${ }^{3}$ So, many studies have been done on the interfacial adhesion between reinforcing fiber and matrix polymer in composite. ${ }^{8-10}$ However, most of the studies on the interfacial adhesion between reinforcing fiber and matrix polymer mainly focused on the system of thermosetting resin and inorganic fiber. Until now, there are very few attempt to evaluate the interfacial adhesion between TLCP and thermoplastics matrix ${ }^{11,12}$ in spite of its practical importance.

The interfacial adhesion between reinforcing fiber and matrix polymer in fiber reinforced composites is affected by many factors such as wettability, residual stress, chemical bond, and welding condition, i.e., welding temperature and time. ${ }^{11-13}$

Therefore, in this study, the interfacial adhesion between TLCP fiber and ductile thermoplastic matrix such as polycarbonate (PC), polyethersulfone (PSF), and polyetherimide (PEI) were evaluated using a single fiber composite (SFC) test. ${ }^{14}$ The main factors affecting the interfacial adhesion in each system are also investigated through the measurement of contact angle, examination of interface, and calculation of the residual stress.

\section{THEORETICAL CONSIDERATION AND DATA ANALYSIS}

In the single fiber composite, the shear in the matrix transfers to the fiber in tensile load through the interface between the fiber and matrix as the specimen is strained. Using a matrix polymer which has a higher strain to failure than the fiber, the fiber breaks repeatedly with increasing strain at its weak point. At some strained state, the fragmentation of fiber ceases although the strain increases continuously, because the fragment length of fibers is short so that the shear transfer along their length can not build up. The critical length, $l_{\mathrm{C}}$ is defined as the fragment length of the fiber when the stress transferred by the matrix is equal to the breaking stress of the fiber. According to Kelly and Tyson, ${ }^{14}$ the critical length of the fiber is given by the following equation.

$$
l_{\mathrm{C}}=\sigma_{\mathrm{f}} d / 2 \tau
$$

where $\sigma_{\mathrm{f}}$ is the breaking stress of the fiber at the critical length, $d$ is the diameter of the fiber, and $\tau$ is the interfacial shear stress between fiber and matrix. Since the distribution of the fragment lengths ranges from $0.5 l_{\mathrm{C}}$ to $1.0 l_{\mathrm{C}}$, the value of $l_{\mathrm{C}}$ is approximated by eq $2 .{ }^{15}$

$$
l_{\mathrm{C}}=4 l_{\mathrm{f}} / 3
$$

where $l_{\mathrm{f}}$ is the mean fragment length.

In order to evaluate the interfacial shear stress, it is important to know the breaking stress, $\sigma_{\mathrm{f}}$ in eq 1 .

\footnotetext{
† To whom all correspondence should be addressed (Tel: +82-562-279-2270; Fax: +82-562-279-2699; e-mail: kwcho@vision.postech.ac.kr).
} 
Weibull distribution function, $G(\sigma)$ which relates the cumulative probability of failure to the applied stress and fiber length, allows the calculation of the fiber stress, $\sigma$ as a function of length of the fiber in accordance with eq 3 and $4 .^{14}$

$$
\begin{aligned}
& G(\sigma)=1-\exp \left[-l(\sigma / \beta)^{\alpha}\right] \\
& \sigma(l)=\left(l \beta / l^{1 / \alpha}\right) \Gamma(1+1 / \alpha)
\end{aligned}
$$

where $\alpha$ is the shape parameter, $\beta$ is the scale parameter, and $\Gamma(1+1 / \alpha)$ is the Gamma function. Equation 3 is also expressed by eq 5 .

$$
\ln [-\ln (1-G)]=\ln \left[l(1 / \beta)^{\alpha}\right]+\alpha \ln \sigma
$$

The $\alpha$ and $\beta$ can be obtained from the intercept and slope in plot of $\ln [-\ln (1-G)]$ against $\ln \sigma$.

\section{EXPERIMENTAL}

\section{Materials and Fiber Spinning}

TLCP used in this study was LC5000 (polyethylene terephthalate $20 \mathrm{~mol} \% / p$-hydroxy benzoic acid $80 \mathrm{~mol} \%$ ) which was obtained from Unitika. Polycarbonate (PC, Lexane 141, G.E.), polyethersulfone (PSF, Udel P1700, Amoco), and polyetherimide (PEI, Ultem 1000, G.E.) were used as a matrix.

The fiber spinning of TLCP was performed using a capillary rheometer (Instron 4204) provided with a spinning unit in condition that the spinning temperature was $285^{\circ} \mathrm{C}$ and extrusion rate was $25 \mathrm{~mm} \mathrm{~min}^{-1}$. The diameter of the fiber was about $40 \mu \mathrm{m}$.

\section{Preparation of Single Fiber Composite}

The thermoplastics such as PC, PSF, and PEI were compression molded using a hot press. The groove in the center of dog-bone shaped sheet was made by razor blade. The TLCP fiber was inserted in the groove of dog-bone shaped sheet and covered with the other sheet. And the welding was performed at welding temperature below $265^{\circ} \mathrm{C}$, the melting temperature of LC5000. The plates were pressed to such an extent that the TLCP fiber was not fractured during welding. The welding temperature and the welding time were varied to investigate the effects of welding condition on the interfacial adhesion.

\section{Measurement of Interfacial Shear Stress}

In order to determine Weibull parameters, $\alpha$ and $\beta$, tensile tests of the TLCP fiber were performed using a universal test machine (Instron 4206) with a cross-head speed of $5 \mathrm{~mm} \mathrm{~min}^{-1}$. The gauge length was $10 \mathrm{~mm}$. The large scattering in the fracture stress and elongation of TLCP fiber occurred due to the defect distributed along the fiber. So, hundred specimens of single fiber were used to obtain a good distribution of fracture stress. The fracture stress was obtained by tensile test and the Weibull distribution function, $G(\sigma)$ of TLCP fiber was obtained through the ratio of the number of fracture at each fracture stress to hundred tests. Weibull parameters were calculated from eq 5 .

The SFC specimens were strained uniaxially up to the point that the fragmentation of the TLCP fiber ceased using a miniature tensile tester installed on optical microscope (Zeiss). The fragment lengths of the TLCP fiber

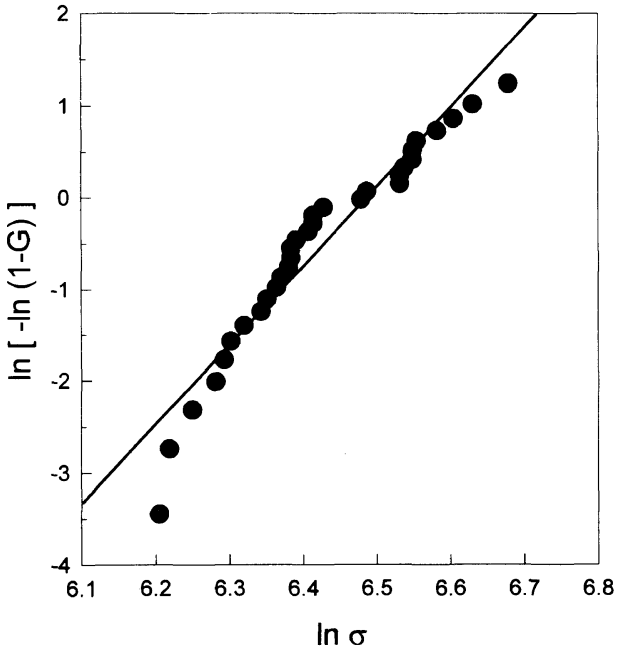

Figure 1. Weibull plot for the LC5000 fiber to calculate Weibull parameters.

were measured by optical microscope and then the mean fragment length, $l_{\mathrm{f}}$ was calculated. The critical length, $l_{\mathrm{C}}$ in eq 1 was obtained from eq 2 . The breaking stress of the fiber at critical length, $\sigma_{\mathrm{f}}$ was calculated using eq 4 and then the interfacial shear stress, $\tau$ was obtained from eq 1 .

\section{Measurement of Contact Angle}

The contact angles of materials were measured using a contact angle meter (Kyowa Kaimenkagaku Co., Ltd.) at $25^{\circ} \mathrm{C}$ in order to calculate the polarity of materials. The water and the diiodomethane were used as reference liquids. The films for measurement of contact angle were prepared by solution casting. The solvents were dichloromethane for PC and PEI, N,N-dimethylformamide for PSF, and $p$-chlorophenol for LC5000.

\section{Observation of Failure Mode at Interface}

After SFC test, the interface in each system was observed to confirm the failure mode using an optical microscope (Zeiss) with a cross-polarizer.

\section{RESULTS AND DISCUSSION}

\section{Fiber Characterization and Interfacial Shear Stress}

Figure 1 shows Weibull plot for the TLCP fiber in terms of the fiber fracture stress and the fracture probability. The data fitted well a straight line, although some data deviated from the linearity. Weibull parameters, $\alpha$ and $\beta$ were calculated from the slope and the intercept in accordance with eq 5 . The calculated values of $\alpha$ and $\beta$ were 8.4 and 871 , respectively.

In the single fiber composite test of matrix/LC5000, the fragmentation of LC5000 fiber occurred mainly in the necked yielding region of the matrix. After the neck propagated over all of the specimen, the fiber fragmentation was complete. The mean fiber fragment aspect ratio as a function of welding time at different welding temperature in matrix/LC5000 is shown in Figure 2. The mean fiber fragment aspect ratio was defined as a ratio of the mean fiber fragment length, $l_{\mathrm{f}}$ measured by optical microscope after test to the diameter of fiber.

In Figure 2, the mean fiber fragment aspect ratio 
K. Cho, T. Kong, and D. LeE

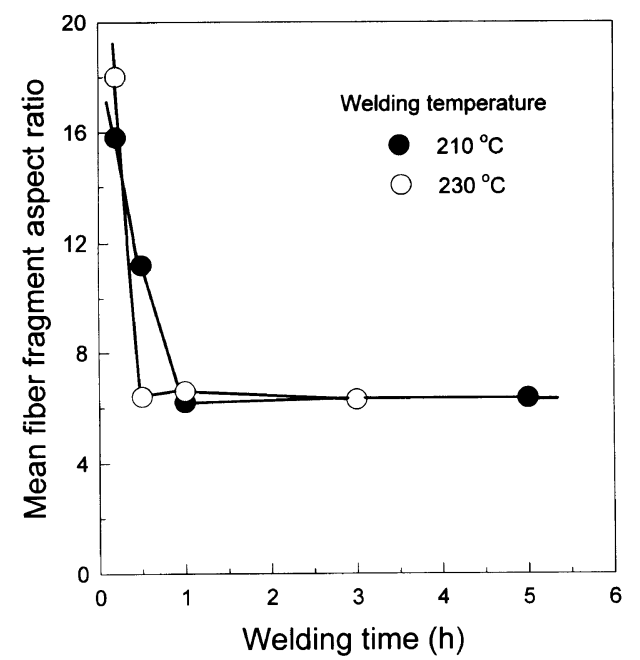

(a)

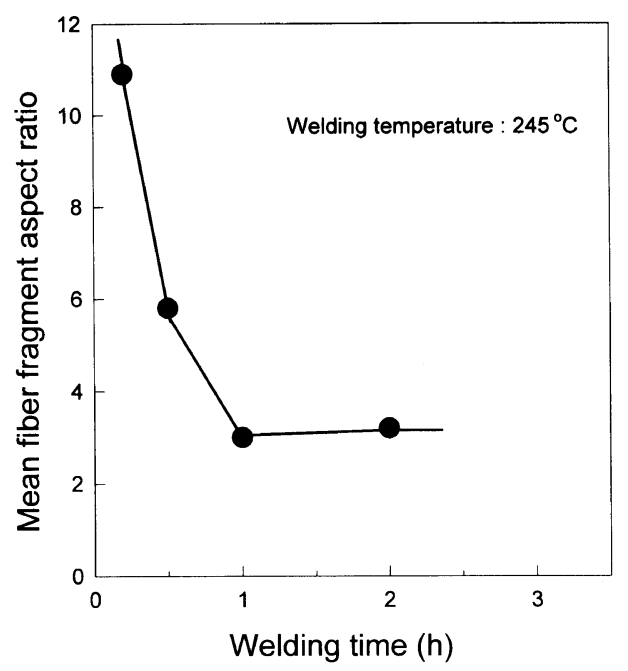

(b)

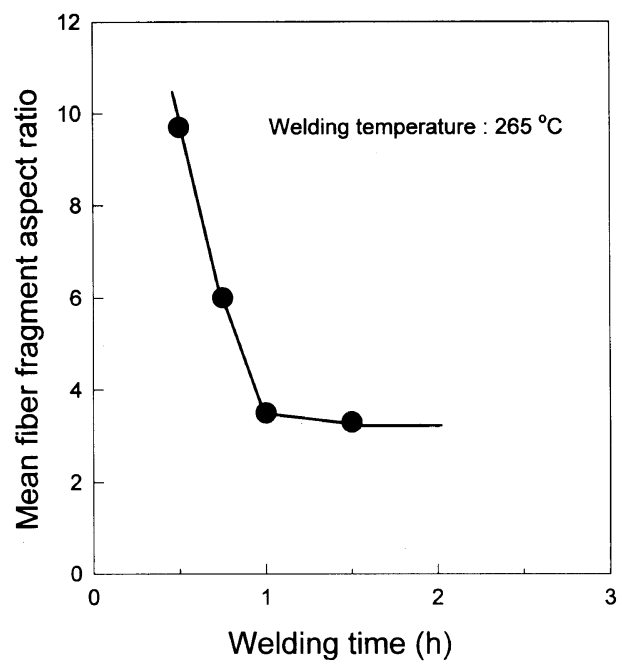

(c)

Figure 2. Mean fiber fragment aspect ratio as a function of welding time for (a) PC/LC5000, (b) PSF/LC5000, and (c) PEI/LC5000.

decreased abruptly at the initial welding time and then became a saturation value in all system. The saturation values of the mean fiber fragment aspect ratio were

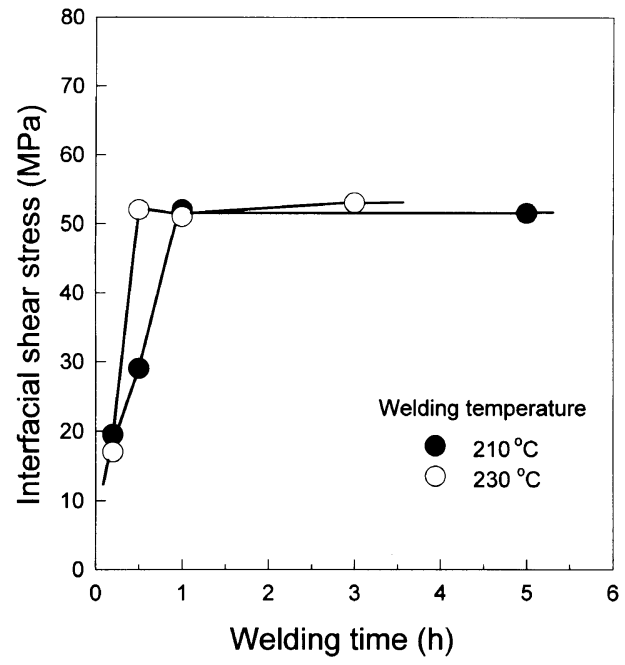

(a)

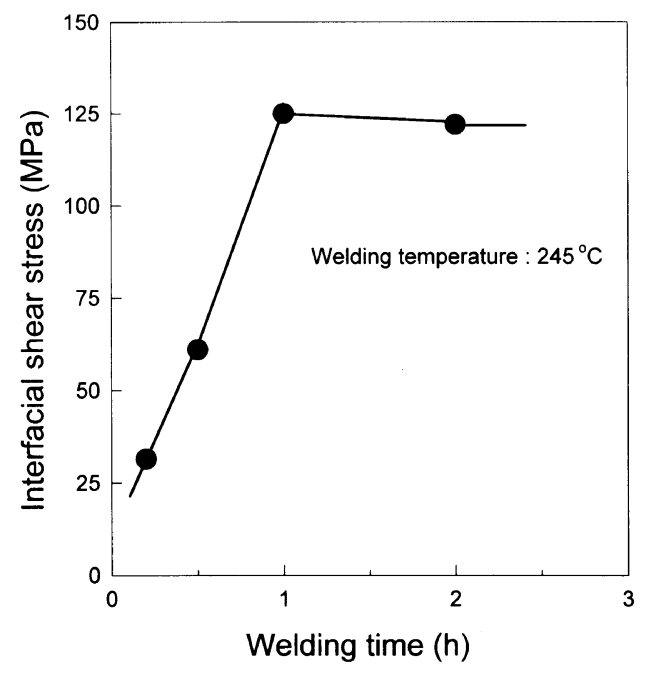

(b)

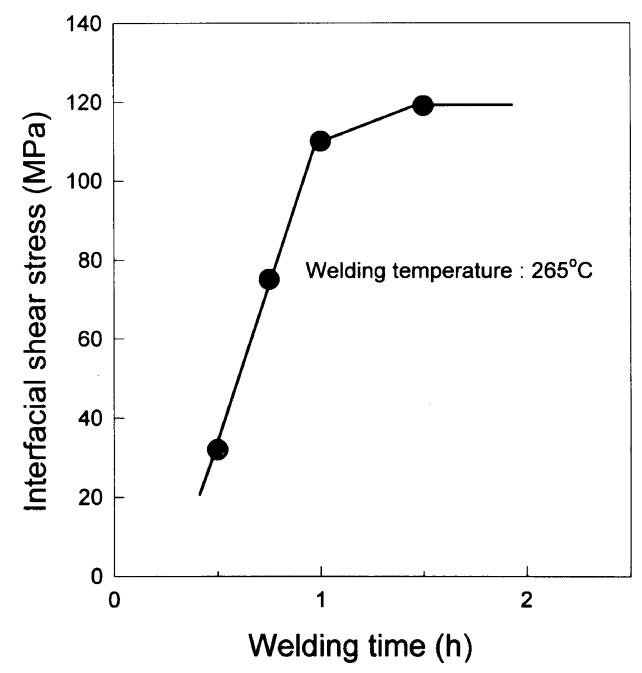

(c)

Figure 3. Interfacial shear stress as a function of welding time for (a) PC/LC5000, (b) PSF/LC5000, and (c) PEI/LC5000.

almost same in different welding temperature in $\mathrm{PC} /$ LC5000, however, the time to the saturation value at $230^{\circ} \mathrm{C}$ was shorter than that at $210^{\circ} \mathrm{C}$. Considering the

Polym. J., Vol. 29, No. 11, 1997 
same saturation value at different welding temperature in PC/LC5000, it was certain that the transesterification reaction between PC and LC5000 did not proceed during welding process. The phenomenon in Figure 2 was attributed to the difference in the wetting of matrix polymer on LC5000 fiber. The wetting of matrix polymer on LC5000 fiber was incomplete at the initial welding time. However, the further wetting proceeded with increasing welding time and then approached the equilibrium. In the case of PC/LC5000 (Figure 2a), the welding time to approach the equilibrium wetting at high welding temperature was shorter than at low welding temperature because of increasing chain mobility of the matrix polymer.

The interfacial shear stress between matrix polymers and LC5000 calculated from eq 1 as a function of welding time at different welding temperature are shown in Figure 3. The interfacial shear stress increased with increasing welding time and then became an equilibrium value. And the welding time to reach the equilibrium value in PC/LC5000 was short in the case of high welding temperature, however, the equilibrium interfacial shear stress, $\tau$ was same regardless of welding temperature. This phenomenon was due to the difference in wetting of matrix polymer on LC5000 fiber with welding time as explained before.

The equilibrium interfacial shear stress in PC/LC5000 was estimated to be $52 \mathrm{MPa}$. Compared this value with the previous work ${ }^{11}$ on PC/LC3000 (polyethylene terephthalate $40 \mathrm{~mol} \% / p$-hydroxy benzoic acid $60 \mathrm{~mol} \%$ ) in which the interfacial shear stress is $33 \mathrm{MPa}$, the equilibrium interfacial shear stress in this study was high considering a similar chemical structure of liquid crystalline polymer in both studies. This different interfacial shear stress in both studies might be due to the difference in welding condition. The welding temperature was higher than that of previous work and the wetting approached the equilibrium value in this study. On the other hand, the welding condition in the previous work was $160^{\circ} \mathrm{C}$ for $6 \mathrm{~h}$. Therefore, the wetting in this study was more complete compared with the previous work and then the higher interfacial shear stress was obtained.

The equilibrium interfacial shear stress in PSF/LC5000 and PEI/LC5000 was $123 \mathrm{MPa}$ and $115 \mathrm{MPa}$, respectively. From the results of measurement of the interfacial shear stress between various matrix polymers and LC5000 using a single fiber composite method, it was found that the equilibrium interfacial shear stress decreased in order of PSF/LC5000, PEI/LC5000, and PC/LC5000.

\section{Effect of Compressive Pressure}

It is known that the interfacial shear stress between reinforcing fiber and matrix polymer was affected by many factors such as compressive pressure due to heat shrinkage, difference in polar interaction, and difference in modulus between reinforcing fiber and matrix. ${ }^{11-13}$ Therefore, the main factors affecting the interfacial shear stress in this study were investigated.

The compressive pressure, $P$ is expressed by the following equation. ${ }^{16}$

$$
P=\left(\alpha_{\mathrm{m}}-\alpha_{\mathrm{f}}\right) \delta T E_{\mathrm{m}} /\left(1+v_{\mathrm{m}}\right)=\alpha_{\mathrm{g}, \mathrm{m}} \delta T E_{\mathrm{m}} /\left(1+v_{\mathrm{m}}\right)
$$

where $\alpha$ is the coefficient of linear thermal expansion, $E$
Table I. Tensile modulus $\left(E_{\mathrm{m}}\right)$, glass transition temperature $\left(T_{\mathrm{g}}\right)$, and coefficient of linear thermal expansion $\left(\alpha_{g}\right)$ of matrix polymers

\begin{tabular}{lccc}
\hline & $E_{\mathrm{m}} / \mathrm{MPa}$ & $T_{\mathrm{g}} /{ }^{\circ} \mathrm{C}$ & $\alpha_{\mathrm{g}} / \mathrm{K}^{-1} \times 10^{-4 \mathrm{a}}$ \\
\hline PC & 13.5 & 145 & 1.22 \\
PSF & 19.6 & 190 & 1.10 \\
PEI & 29.4 & 214 & 1.05 \\
\hline
\end{tabular}

${ }^{\text {a }}$ Calculated by using Simha-Boyer relationship and Boyer-Spencer correction. ${ }^{17}$

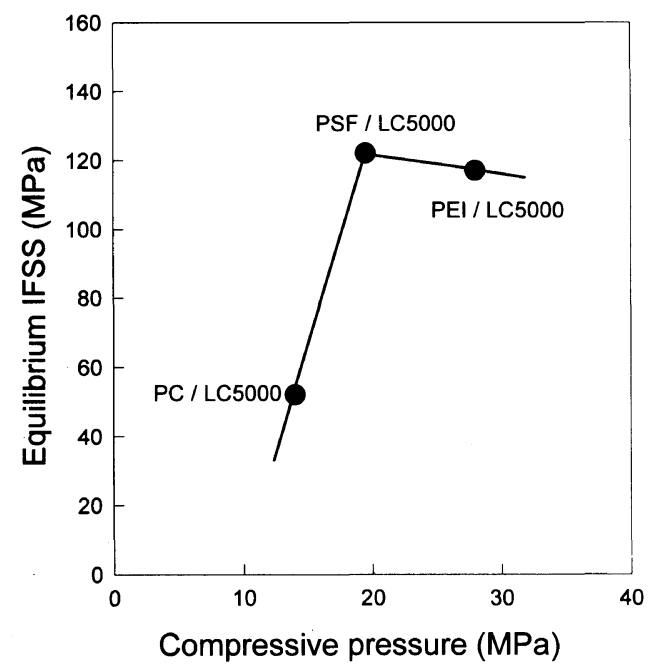

Figure 4. Equilibrium interfacial shear stress, $\tau$ as a function of compressive pressure, $P$.

is the tensile modulus, $v$ is Poisson's ratio, and $\delta T$ is the temperature difference between the glass transition temperature and room temperature. The subscripts, $\mathrm{m}$, $\mathrm{f}$, and $\mathrm{g}$ indicate the matrix polymer, fiber, and glassy state, respectively. The tensile modulus, $E_{\mathrm{m}}$ was measured using a universal test machine with a cross-head speed of $5 \mathrm{~mm} \mathrm{~min}^{-1}$. The coefficient of linear thermal expansion $\alpha_{\mathrm{g}}$ was calculated using Simha-Boyer relationship and Boyer-Spencer correction. ${ }^{17}$ The coefficient of linear thermal expansion, tensile modulus, and the glass transition temperature used to calculate the compressive pressure in eq 6 are shown in Table I and Poission's ratio was taken as 0.35 .

The equilibrium interfacial shear stress as a function of the compressive pressure in each system is shown in Figure 4 . The linear relationship was not obtained between the equilibrium interfacial shear stress and the compressive pressure. The equilibrium interfacial shear stress in PSF/LC5000 was unusually high and deviated severely from the linear line. Therefore, it was speculated that the other factors besides the compressive pressure were involved in the development of interfacial shear stress.

\section{Effect of Polarity Difference}

It was supposed that the polar interaction between matrix polymers and LC5000 fibers affected the interfacial shear stress because of the polarity difference of materials in this study. Thus, the polarity was determined using surface tension which was calculated from the contact angle in accordance with Young's equa- 


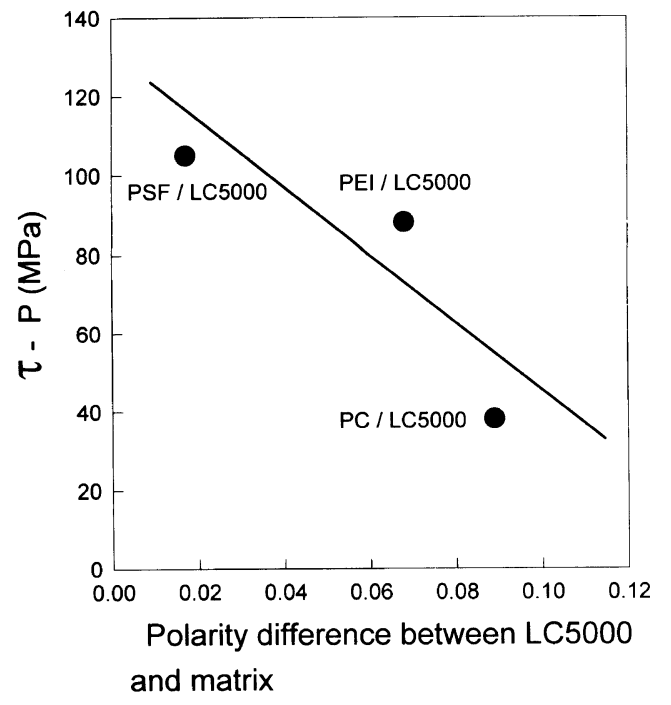

Figure 5. Plot of $\tau-P$ against difference in the polarity between matrix polymers and LC5000 fiber.

tion and Harmonic-Mean equation. ${ }^{18}$ The polarity is defined as the fraction of polar contribution to surface tension. The method of polarity measurement was described in detail elsewhere. ${ }^{18}$

The effect of polarity difference between matrix polymers and LC5000 is shown in Figure 5. In order to exclude the effect of compressive pressure and evaluate only the effect of polarity on the interfacial shear stress, the difference between equilibrium interfacial shear stress and compressive pressure, $\tau-P$ was considered and taken as a vertical axis. It was found that the interfacial shear stress excluding the effect of compressive pressure increased as the difference in the polarity decreased. The high value of the equilibrium interfacial shear stress of PSF/LC5000 as shown in Figure 4 disappeared and a relatively linear relation existed between the polarity difference and $\tau-P$. Therefore, in the case of PSF/LC5000, the polar interaction between PSF and LC5000 affected the equilibrium interfacial shear stress besides the compressive pressure. On the other hand, the equilibrium interfacial shear stress might be explained in terms of the compressive pressure in the case of PC/LC5000 and PEI/LC5000.

\section{Failure Mode}

After the single fiber composite test, the interface between matrix polymer and LC5000 fiber was examined using optical microscope to investigate the failure mode. It is well known that there are three failure modes in composite, i.e., debonding at the interface, shear deformation of the matrix, and transverse matrix crack. The failure mode changes from the debonding at the interface to the transverse matrix crack as the interfacial adhesion strength increases. ${ }^{13}$ The optical micrographs of interface in each system after a single fiber composite test are shown in Figure 6. In the case of PC/LC5000 (Figure 6a), the debonding at the interface between PC and LC5000 fiber occurred and the shear deformation of PC was not observed, which was the evidence of the poor adhesion between PC and LC5000 fiber. On the other hand, in the case of PSF/LC5000 (Figure 6b), the plastic deformation of the matrix polymer adjacent to

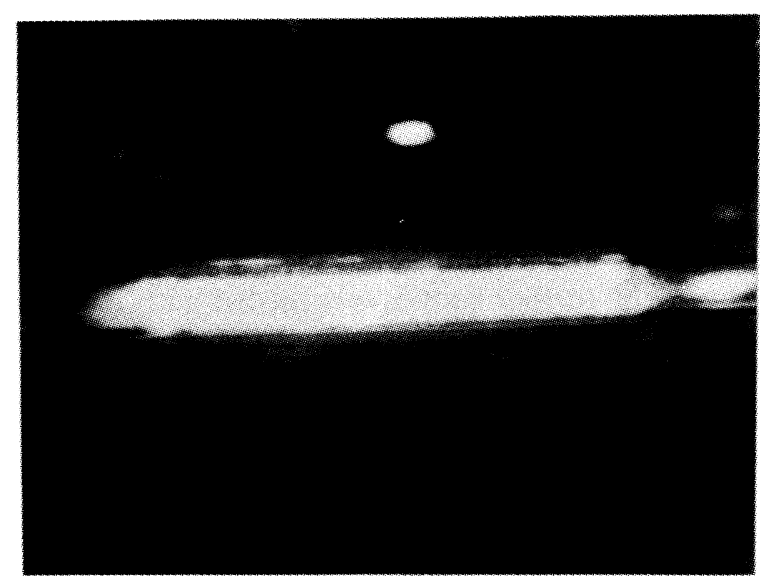

(a)

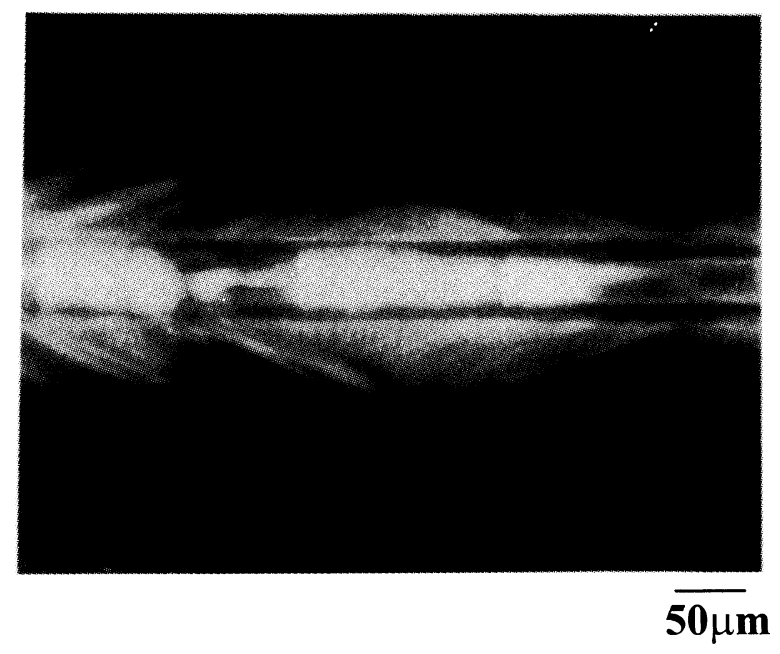

(b)

Figure 6. Optical micrographs of interface after single fiber composite test for (a) PC/LC5000 and (b) PSF/LC5000.

the interface was observed. Thus, the good interfacial adhesion in PSF/LC5000 was also confirmed by observation of failure mode and this result is consistent with the values of equilibrium interfacial shear stress as already shown in Figure 3.

\section{CONCLUSION}

The interfacial shear stress between LC5000 fiber and various thermoplastics such as PC, PSF, and PEI was measured using a single fiber composite test. The interfacial shear stress increased abruptly as welding time increased at fixed welding temperature and became an equilibrium value in each system. It was attributed that the wetting of matrix polymer on LC5000 fiber became complete as the welding time increased. The equilibrium interfacial shear stress, $\tau$ decreased in the order of PSF/ LC5000, PEI/LC5000, and PC/LC5000 system. The optical micrograph showed that the debonding occurred at the interface and the plastic deformation of the matrix was not observed in the case of PC/LC5000. However, the plastic deformation of the matrix near the interface was observed in the case of PSF/LC5000. These results were consistent with the values of equilibrium interfacial shear stress. From the results of equilibrium interfacial 
shear stress and other measurements, such as compressive pressure and polarity difference between LC5000 fiber and thermoplastics, polar interaction and compressive pressure due to thermal shrinkage were found to be major factors governing the equilibrium interfacial shear stress.

Acknowledgment. This work was supported by Non Directed Research Funds from the Korea Research Foundation, 1996.

\section{REFERENCES}

1. M. Kimura and R. S. Porter, J. Polym. Sci., Polym. Phys. Ed., 22, 1697 (1984).

2. E. G. Joseph, G. L. Wilkes, and P. G. Baird, "Polymer Liquid Crystals," Plenum Press, New York, N.Y., 1984.

3. B. R. Basset and A. F. Yee, Polym. Compos., 11, 10 (1990).

4. S. H. Jung and S. C. Kim, Polym. J., 20, 73 (1988).

5. P. Zhuang, T. Kyu, and J. L. White, Polym. Eng. Sci., 28, 1095
(1988).

6. S. Mehta and B. L. Deopura, J. Appl. Polym. Sci., 56, 169 (1995).

7. S. Mehta and I. Isayev, Polym. Eng. Sci., 31, 9 (1991).

8. V. Rao and L. T. Drzal, Polym. Compos., 12, 48 (1991).

9. B. Yavin, H. E. Gallis, J. Scherf, and A. Eitan, Polym. Compos., 12, 436 (1991)

10. J. M. Park, E. M. Chong, W. G. Shin, S. I. Lee, D. J. Yoon, and J. H. Lee, Polymer (Korea), 20, 753 (1996)

11. C. Carfagna, P. A. Netti, and L. Nicolais, Polym. Compos., 13, 169 (1992)

12. S. G. James, A. M. Donald, I. S. Miles, L. Mallagh, and W. A. MaCdonald, J. Polym. Sci., Polym. Phys. Ed., 31, 221 (1993).

13. E. V. Pisanova, S. F. Zhandraov, and V. A. Dovgyalo, Polym. Compos., 15, 147 (1994).

14. A. Kelly and W. R. Tyson, J. Mech. Phys. Solids, 13, 329 (1965).

15. A. S. Wimolkitatisak and J. Bell, Polym. Compos., 10, 162 (1989).

16. T. Ohsawa, A. Nakayama, M. Miwa, and A. Hasegawa, J. Appl. Polym. Sci., 22, 3203 (1978).

17. J. Bicerano, "Prediction of Polymer Properties," Marcel Dekker, New York, N.Y., 1993.

18. S. Wu, "Polymer Interface and Adhesion," Marcel Dekker, New York, N.Y., 1982. 